\title{
Impuesto sobre la renta de las personas físicas y desigualdad de los ingresos en el Ecuador entre 2007 y 2011
}

\author{
Liliana Cano
}

\section{Resumen}

En este trabajo se analiza el efecto redistributivo del impuesto sobre la renta de las personas físicas en el Ecuador. En primer lugar, se calculan los índices de progresividad y el efecto redistributivo de este impuesto. Luego, mediante técnicas de microsimulación, se estudia su efecto redistributivo bajo distintas hipótesis de ingreso imponible. Finalmente, se calculan los tipos impositivos efectivos pagados por los grupos de ingresos más altos y se obtiene una gama de impuestos sobre la renta óptimos para el grupo de ingresos correspondiente al 1\% más rico de la población. Los resultados muestran, en primer lugar, que las personas de altos ingresos tienen mayor probabilidad de reducir su ingreso imponible mediante deducciones fiscales legales con respecto a las personas de bajos ingresos. En segundo lugar, mientras los tipos impositivos efectivos pagados por las personas de altos ingresos son relativamente bajos, los tipos impositivos óptimos podrían llegar hasta el 63\%.

\section{Palabras clave}

Impuesto a la renta, política fiscal, distribución del ingreso, modelos matemáticos, métodos de simulación, Ecuador

\section{Clasificación JEL}

D31, H24, O54

\section{Autora}

Liliana Cano es Investigadora posdoctoral del Laboratorio de Estudios e Investigación sobre Economía, Política y Sistemas Sociales (LEREPS) de la Universidad de Toulouse 1 Capitole, Francia. Correo electrónico: liliana.cano@ut-capitole.fr. 



\section{Introducción}

El interés en el estudio de la desigualdad de los ingresos ha crecido en los últimos años, tanto en el ámbito de la investigación como en el de la política. Desde los aportes seminales de Piketty (2001 y 2014), Piketty y Saez (2003) y Atkinson y Piketty (2007 y 2010) sobre la evolución a largo plazo de la desigualdad de los ingresos y de la riqueza en la mayoría de los países industrializados, el debate público reciente se ha concentrado en el papel de los impuestos sobre la renta en la reducción de la desigualdad (Atkinson, 2014; Piketty, 2015). El impuesto sobre la renta de las personas físicas es el instrumento de política pública que a menudo se tiene en cuenta cuando el principal objetivo es modificar la distribución del ingreso después de impuestos (Poterba, 2007). Sin embargo, la posibilidad de reducir la desigualdad de los ingresos mediante la tributación depende fundamentalmente del grado de progresividad de los impuestos del país de que se trate. Así, el efecto redistributivo de los impuestos sobre la renta se ha convertido en un tema central, tanto en los países desarrollados como en los países en desarrollo.

Este trabajo arroja nueva luz sobre el efecto redistributivo del impuesto sobre la renta de las personas físicas en el Ecuador entre 2007 y 2011. Con arreglo a la práctica común en la literatura sobre evaluación de políticas públicas, en primer lugar se calculan diferentes índices de progresividad y efecto redistributivo de los impuestos, a saber, los índices de Kakwani, Suits y Reynolds-Smolensky. En segundo lugar, se emplean técnicas de microsimulación para simular el efecto redistributivo del impuesto sobre la renta de las personas físicas del Ecuador en el marco de distintas hipótesis de ingreso imponible. Se consideran diferentes definiciones de deducciones del impuesto sobre la renta y se presentan hipótesis alternativas que podrían mejorar el efecto redistributivo de dicho impuesto. En tercer lugar, conforme la literatura sobre los altos ingresos (Piketty, 2001; Piketty y Saez, 2003; Atkinson y Piketty, 2010), se utilizan series homogéneas de participación de los altos ingresos en el ingreso total en el Ecuador de 2007 a 2011 (Cano, 2015) para calcular los tipos impositivos efectivos pagados por los grupos de ingresos más altos. Por último, de acuerdo con la literatura sobre elasticidad del ingreso imponible (Lindsey, 1987; Feldstein, 1999; Auten y Carroll, 1999; Gruber y Saez, 2002; Saez, 2001; Chetty, 2009; Saez, Slemrod y Giertz, 2012), y utilizando diferentes valores de elasticidades compensadas y no compensadas, se obtiene una gama de tipos impositivos óptimos para el grupo de ingresos correspondiente al 1\% más rico de la población. Los resultados se basan en datos de declaraciones de la renta individuales compiladas anualmente por el Servicio de Rentas Internas del Ecuador.

El estudio del efecto redistributivo del impuesto sobre la renta de las personas físicas en el Ecuador aquí realizado obedece a dos motivos principales. En primer lugar, en varios estudios recientes se ha documentado la disminución de la desigualdad de los ingresos en la mayoría de los países latinoamericanos desde inicios de la década de 2000 (Gasparini y otros, 2009; Cornia, 2010; López-Calva y Lustig, 2010; Lustig, López-Calva y Ortiz-Juárez, 2013; Cornia, 2014; CEPAL, 2012, 2013 y 2014), sobre todo debido a: i) una reducción de la prima por calificación, ii) una disminución de la desigualdad salarial entre las zonas urbanas y rurales, iii) programas de transferencias públicas como los de transferencias monetarias condicionadas y iv) condiciones externas favorables. Además, mientras que la tributación tuvo un efecto insignificante en la desigualdad de los ingresos en décadas anteriores (Cornia, Gómez Sabaini y Martorano, 2011), en un número cada vez mayor de estudios se documenta el efecto positivo de las reformas tributarias de la década de 2000 en la reducción de la desigualdad de los ingresos en la región (Jiménez, Gómez Sabaini y Podestá, 2010; Roca, 2009; Cetrángolo y Gómez Sabaini, 2006; Cornia, Gómez Sabaini y Martorano, 2011; Hanni, Martner y Podestá, 2015). Con este trabajo se procura contribuir a esa literatura mediante la evaluación del efecto redistributivo del impuesto sobre la renta de las personas físicas en el Ecuador.

Junto con el surgimiento a fines de la década de 1990 de los programas de transferencias monetarias condicionadas, que promueven la acumulación de capital humano a largo plazo entre los 
menos favorecidos (Rawlings y Rubio, 2005), los encargados de la formulación de políticas en América Latina y el Caribe se han concentrado en mejorar la utilización de los programas de transferencias públicas para lograr una distribución del ingreso más justa (por ejemplo, perfeccionando la fijación de objetivos e incrementando la cobertura). Es probable que el papel de los impuestos para enfrentar la desigualdad de los ingresos se haya relegado a un segundo plano porque los sistemas tributarios latinoamericanos se han basado sobre todo en impuestos indirectos (impuesto sobre el valor agregado e impuestos comerciales), pese a que la tributación progresiva sobre la renta comenzara a introducirse en la región a comienzos del siglo XX (Gómez Sabaini, 2006; Cornia, Gómez Sabaini y Martorano, 2011). Sin embargo, el debate de los últimos años en América Latina se ha orientado cada vez más a la necesidad de reforzar la progresividad tributaria e incrementar la recaudación tributaria para, de ese modo, mejorar las transferencias públicas. De hecho, las reformas sociales, económicas e institucionales realizadas en la mayor parte de América Latina y el Caribe desde comienzos de la década de 2000 han dado lugar a una nueva ola de reformas tributarias ${ }^{1}$. De acuerdo con Fairfield (2010) y Gómez Sabaini y Moran (2013), esta puede caracterizarse como una "segunda generación" de reformas tributarias dirigidas a: i) mejorar la recaudación tributaria y la equidad del sistema tributario, ii) incrementar la progresividad tributaria y iii) fortalecer la capacidad de las agencias tributarias para combatir la evasión. Así, la tributación progresiva y la redistribución del ingreso han sido los objetivos primarios de la política tributaria latinoamericana en los últimos años, por lo menos en teoría (Cornia, Gómez Sabaini y Martorano, 2011).

No obstante los considerables avances realizados para lograr estos objetivos, aún persisten retos y trabajo por hacer, pues los sistemas tributarios todavía se basan en impuestos indirectos, que determinan que todo el sistema sea muy regresivo². Goñi, López y Servén (2011) muestran, por ejemplo, que la desigualdad de los ingresos después de impuestos y transferencias ha disminuido menos en América Latina y el Caribe que en los países de la Organización de Cooperación y Desarrollo Económicos (OCDE), mientras que el coeficiente de Gini antes de la política fiscal es casi el mismo en los dos grupos de países. Los autores también muestran que la reducción de la desigualdad de los ingresos en América Latina y el Caribe se ha logrado principalmente mediante transferencias públicas y que los impuestos han sido poco útiles para hacer frente a la desigualdad. El sistema tributario del Ecuador no es una excepción ${ }^{3}$. La reforma tributaria de 2008 se promulgó precisamente para incrementar la progresividad del impuesto sobre la renta de las personas físicas, promover la equidad tributaria y aumentar la recaudación. Esto supuso, en primer lugar, la creación de dos categorías adicionales de impuesto sobre la renta y un tipo impositivo marginal máximo del 35\% y, en segundo lugar, la introducción de nuevas deducciones del impuesto sobre la renta de las personas físicas para gastos en vivienda, educación, salud, vestimenta y alimentos ${ }^{4}$. Sin embargo, todavía se sabe muy poco sobre los efectos de esta reforma tributaria en la desigualdad de los ingresos en el Ecuador.

\footnotetext{
1 Véase una revisión detallada de las reformas y los patrones de política tributaria en América Latina en Gómez Sabaini (2006), Cetrángolo y Gómez Sabaini (2006), González y Martner (2009), Jiménez, Gómez Sabaini y Podestá (2010), Gómez Sabaini y Jiménez (2012), Tanzi (2013) y Gómez Sabaini y Moran (2013).

2 En los informes técnicos elaborados por la Comisión Económica para América Latina y el Caribe (CEPAL) se documentan los principales logros de las últimas reformas tributarias en América Latina y el Caribe, al igual que en el último informe sobre estadísticas de ingresos en América Latina producido conjuntamente por la Organización de Cooperación y Desarrollo Económicos (OCDE), el Centro Interamericano de Administraciones Tributarias (CIAT), el Banco Interamericano de Desarrollo (BID) y la CEPAL.

3 En el Ecuador, los ingresos obtenidos mediante impuestos indirectos como el impuesto sobre el valor agregado (IVA) representaron en promedio el 4,9\% del producto interno bruto (PIB) en el período 1990-2000 y aumentaron a casi el 8\% del PIB en 2001-2013. En 2013, además, el 53\% de la recaudación tributaria total correspondió a impuestos indirectos y poco menos del $21 \%$ a impuestos sobre la renta, beneficios y ganancias de capital (OCDE/CEPAL/CIAT/BID, 2015).

${ }^{4}$ Las deducciones del impuesto sobre la renta de las personas físicas se introdujeron en la reforma tributaria de 2008 para promover la equidad tributaria. El principal objetivo era precisamente permitir que los contribuyentes de bajos ingresos se beneficiaran de mayores deducciones fiscales.
} 
El segundo motivo de este trabajo fue el creciente interés en el estudio de la participación de los altos ingresos en el ingreso total y los tipos impositivos efectivos pagados por las personas de altos ingresos. Después de los aportes seminales de Piketty (2001 y 2014) y Piketty y Saez (2003) sobre la distribución a largo plazo de los ingresos más altos en Francia y los Estados Unidos, la relación entre la concentración del ingreso y la política tributaria ha recibido considerable atención, tanto el ámbito de la investigación como en el de la política. Conforme esa literatura, Cano (2015) construyó series anuales de participación de los altos ingresos en el Ecuador de 2004 a 2011, a partir de datos de declaraciones de la renta individuales y utilizando totales de población e ingresos basados en estimaciones de los censos y las cuentas nacionales, respectivamente, como controles externos. En 2010, la participación del 1\% más rico de la población en el ingreso total variaba entre el 14\% y el $17 \%$, según la manera en que se define el ingreso utilizado como numerador de la proporción ${ }^{5}$. En este trabajo se emplean esas estimaciones para calcular los tipos impositivos efectivos pagados por los grupos de ingresos más altos. Otro motivo fue el creciente interés en el grado de progresividad que los impuestos sobre la renta deberían tener. Utilizando diferentes estimaciones de elasticidad y con arreglo a la literatura sobre elasticidad del ingreso imponible (Saez, 2001; Gruber y Saez, 2002), se obtuvo una gama de tipos impositivos óptimos para el grupo de ingresos correspondiente al 1\% más rico de la población.

El resto de este artículo se organiza de la siguiente manera: en la sección II se describen los datos y la metodología empleados, en la sección III se detallan los resultados y en la sección IV se presentan las conclusiones y se examinan las repercusiones en materia de políticas.

\section{Datos y métodos}

\section{Datos}

Las estimaciones se basan en declaraciones de la renta individuales compiladas anualmente por la administración tributaria ecuatoriana ${ }^{6}$. La información sobre las personas registrada en la base de datos de declaraciones fiscales incluye: i) ingresos laborales en forma de salarios y sueldos, ii) ingresos de capital (dividendos, intereses y otros ingresos de capital), iii) ingresos industriales o comerciales, iv) ingresos por trabajo autónomo, v) ingresos de otras fuentes y vi) deducciones fiscales, obligaciones tributarias e impuestos pagados por los contribuyentes.

Los datos utilizados para el análisis provienen de tres formularios de impuestos: i) el formulario 102, utilizado para declarar información sobre salarios, ingresos por trabajo autónomo, ingresos industriales o comerciales, ingresos de capital y otras posibles fuentes de ingresos de los contribuyentes obligados a llevar contabilidad (por ejemplo, personas que realizan actividades comerciales); ii) el formulario 102A, utilizado para declarar información sobre salarios, ingresos por trabajo autónomo, ingresos de capital y otras posibles fuentes de ingresos de los contribuyentes no obligados a llevar contabilidad; iii) el formulario 107, utilizado para declarar información sobre ingresos del trabajo en relación de dependencia. La renta de las personas físicas se grava a tipos impositivos marginales progresivos que van del $0 \%$ al 35\%. Las personas cuya única fuente de ingresos es el salario (es decir, los empleados) no deben compilar una declaración fiscal porque el impuesto sobre

\footnotetext{
5 Para ofrecer un panorama preciso de los ingresos en la cima de la distribución, Cano (2015) construyó series de participación de los altos ingresos en el ingreso total en el Ecuador utilizando distintos criterios (por ejemplo, ingresos netos, ingreso brutos) y diferentes porcentajes de costos intermedios y deducciones como numeradores de las proporciones. En 2011, el ingreso correspondiente al $1 \%$ más rico de la población variaba entre el $12 \%$ y el $15 \%$.

6 El Servicio de Rentas Internas (SRI) del Ecuador permitió amablemente a la autora el acceso a toda la base de datos de declaraciones de la renta de las personas físicas de 2004 a 2011. La base de datos está compuesta por una media de casi 1,9 millones de observaciones (2,3 millones en 2011). En este estudio se trabaja con el período que va de 2007 a 2011.
} 
la renta es automáticamente retenido por los empleadores. No obstante, los empleados que obtienen ingresos de otras fuentes además de los salarios (dividendos, intereses, rentas, entre otras) deben consolidar todas las fuentes de ingresos (por ejemplo salarios e ingresos de capital) en una única declaración fiscal anual (formulario 102 o 102A). A los ingresos totales se aplica la misma escala tributaria progresiva del $0 \%$ al 35\%. Se identificó a los contribuyentes cuyos ingresos se declararon tanto mediante el formulario 107 como mediante el formulario 102 o 102A para no duplicar los datos sobre salarios y sueldos. En todos esos casos se trabajó solamente con los datos del formulario 102 o 102A. Con respecto a los ingresos de capital, antes de 2010 los dividendos distribuidos que ya se habían gravado con impuestos de sociedades se exoneraron del impuesto sobre la renta de las personas físicas para evitar la doble tributación. Desde 2010, los dividendos recibidos por las personas que viven en el Ecuador forman parte de la base del impuesto sobre la renta de las personas físicas. Los impuestos sobre la renta en el Ecuador se declaran en dólares estadounidenses y se evalúan a nivel individual y no a nivel del hogar como, por ejemplo, en los Estados Unidos y en algunos países europeos como Alemania, Francia y el Reino Unido.

Según los estándares latinoamericanos, la base de datos de las declaraciones fiscales del Ecuador está aproximadamente en un punto medio en cuanto al número de personas cubiertas. Casi el 27\% de la población adulta (20 o más años de edad) declaró sus ingresos a la administración tributaria en 2011, incluidas las personas cuyos ingresos estaban por debajo del umbral impositivo ${ }^{7}$. La comparación con otros países para los cuales se dispone de estimaciones sobre la participación de los altos ingresos y la incidencia de los impuestos revela que ese porcentaje es inferior a la cobertura de la población adulta en Chile (el 67\% en 2009) y el Uruguay (el 74\% en 2012), pero superior a la cobertura en Colombia (el $4 \%$ en 2010) y la Argentina (el 3\% en 2004) ${ }^{8}$.

\section{Métodos}

Para evaluar la relación entre el impuesto sobre la renta de las personas físicas y la desigualdad de los ingresos se utilizan cuatro métodos complementarios. En primer lugar, para analizar el efecto redistributivo de los impuestos sobre la renta se calculan diferentes índices de progresividad y redistribución comúnmente propuestos en la literatura sobre evaluación del impacto: los índices de Kakwani y Suits para medir el nivel de progresividad y el índice de Reynolds-Smolensky para medir el efecto redistributivo del impuesto sobre la renta de las personas físicas en el Ecuador. Al mismo tiempo, se trazan las curvas de concentración para las deducciones del impuesto sobre la renta de las personas físicas y las obligaciones tributarias, junto con la curva de Lorenz de los ingresos antes de impuestos correspondiente a 2008 y 2010. Estos indicadores de progresividad y redistribución permiten estudiar el efecto distributivo de los impuestos sobre la renta y comparar la distribución del ingreso antes y después de impuestos.

Las curvas de concentración y los índices sintéticos de Kakwani, Suits y Reynolds-Smolensky se obtienen a partir del enfoque clásico de la curva de Lorenz y el coeficiente de Gini. La curva de Lorenz $\left(L_{p}\right)$ representa el porcentaje acumulativo de ingresos en el eje vertical en relación con el porcentaje acumulativo de personas, clasificadas según los ingresos de más pobres a más ricas, en el eje horizontal. El coeficiente de Gini $\left(G_{p}\right)$ compara el área entre la curva de Lorenz $\left(L_{p}\right)$ y la diagonal de igualdad perfecta $\left(45^{\circ}\right)$ con el área total debajo de la diagonal, tomando valores que oscilan entre 0 (igualdad absoluta) y 1 (desigualdad absoluta).

\footnotetext{
7 Los datos de la población de 20 o más años de edad provienen de la Encuesta Nacional de Empleo, Desempleo y Subempleo (ENEMDU) del Ecuador realizada en diciembre de 2011.

8 Los estudios sobre la participación de los altos ingresos y la incidencia de los impuestos corresponden a Fairfield y Jorratt (2016) para Chile, Burdín, Esponda y Vigorito (2014) para el Uruguay, Alvaredo y Londoño (2013) para Colombia y Alvaredo (2010) para la Argentina. La baja cobertura de la población en la Argentina y Colombia probablemente se debe a que sus estimaciones no incluyen a los contribuyentes cuya única fuente de ingresos es el salario.
} 
La curva de Lorenz y el coeficiente de Gini pueden reformularse para evaluar cambios en la distribución del ingreso, en particular cuando se incluyen impuestos. La curva de concentración representa el porcentaje acumulativo de obligaciones tributarias en el eje vertical en relación con el porcentaje acumulativo de personas, clasificadas según los ingresos, en el eje horizontal. Si la curva de concentración de los impuestos se encuentra más lejos que la curva de Lorenz con respecto a la diagonal de igualdad perfecta, los impuestos están distribuidos de manera más desigual que los ingresos y, en consecuencia, son progresivos. El coeficiente de concentración correspondiente $C_{t}$, también conocido como coeficiente de cuasi Gini, se interpreta en forma análoga al coeficiente de Gini.

El índice de progresividad de Kakwani se calcula comparando la curva de concentración de los impuestos con la curva de Lorenz de los ingresos antes de impuestos. El índice de Kakwani se define como el doble del área entre la curva de Lorenz de los ingresos antes de impuestos y la curva de concentración de los impuestos. Así, este equivale a la diferencia entre el coeficiente de concentración de los impuestos (o coeficiente de cuasi Gini) y el coeficiente de Gini de la distribución del ingreso antes de impuestos (Kakwani, 1977):

$$
K=C_{t}-G_{y}
$$

donde $C_{t}$ es el coeficiente de concentración de los impuestos (o coeficiente de cuasi Gini) y $G_{y}$ es el coeficiente de Gini de la distribución del ingreso antes de impuestos. Un índice de Kakwani positivo indica que los impuestos son progresivos (es decir, las obligaciones tributarias aumentan con los ingresos), un índice de Kakwani negativo indica que los impuestos son regresivos (es decir, las obligaciones tributarias disminuyen con los ingresos) y un índice de Kakwani igual a 0 indica que los impuestos son proporcionales a los ingresos.

Otro índice de progresividad ampliamente usado es el propuesto por Suits (1977), que mide la desviación de la proporcionalidad al comparar la curva de Lorenz de los ingresos antes de impuestos con la línea diagonal de la igualdad perfecta. El índice de Suits es una adaptación del coeficiente de Gini y se construye representando el porcentaje acumulativo de impuestos en el eje vertical en relación con el porcentaje acumulativo de ingresos en el eje horizontal. Como sugieren Amarante y otros (2011), el índice de Suits se puede formular de la siguiente manera:

$$
S=2 \int_{0}^{1}\left(i-C_{f}(i)\right) d i
$$

Si los impuestos son proporcionales, la curva de concentración de los impuestos coincide con la línea diagonal de igualdad perfecta $\left(45^{\circ}\right)$ y el índice de Suits asume el valor 0 . Si los impuestos son progresivos, la curva de concentración estará por debajo de la línea de igualdad perfecta y el índice de Suits será positivo. Si los impuestos son regresivos, la curva de concentración estará por encima de la línea de igualdad perfecta y el índice de Suits será negativo. Por ejemplo, si solo las personas de altos ingresos pagaran impuestos, el índice de Suits tomaría el valor 1. Por el contrario, si solo las personas más pobres pagaran impuestos, el índice de Suits tomaría el valor -1. Aunque el diseño de los índices de Kakwani y Suits es bastante similar, existen algunas diferencias entre ellos. Como señalan Amarante y otros (2011), mientras el índice de Kakwani se integra con respecto a la población, el índice de Suits se integra con respecto a los ingresos.

Para medir el efecto redistributivo de los impuestos sobre la renta se utiliza el índice de ReynoldsSmolensky, que mide la manera en que los impuestos afectan la distribución del ingreso después de impuestos, captando la diferencia entre los coeficientes de Gini de los ingresos antes y después de impuestos de la siguiente manera:

$$
R S=G_{y}-G_{y-t}
$$


donde $G_{y}$ es el coeficiente de Gini antes de impuestos y $G_{y-t}$ es el coeficiente de Gini después de impuestos (Reynolds y Smolensky, 1977). Un índice de Reynolds-Smolensky positivo indica que los impuestos son progresivos, porque la distribución del ingreso después de impuestos es más equitativa que la distribución del ingreso antes de impuestos. Un valor elevado del índice de ReynoldsSmolensky sugiere que los impuestos tienen un gran potencial redistributivo.

En segundo lugar, se utilizan técnicas de microsimulación estáticas para simular el efecto redistributivo del impuesto sobre la renta de las personas físicas en el Ecuador con diferentes definiciones de ingreso imponible. Para cada observación se simula aritméticamente el ingreso antes de impuestos según dos hipótesis contrarias a los hechos: i) un umbral del 50\% para costos y deducciones y ii) la eliminación de todas las deducciones del impuesto sobre la renta ${ }^{9}$. Estas hipótesis muestran lo que ocurriría si se implementaran cambios en las deducciones del impuesto sobre la renta. A continuación se aplican tipos impositivos y escalas del impuesto sobre la renta en ambas hipótesis y a cada observación para calcular las obligaciones tributarias y los ingresos después de impuestos. Por último, se calculan los índices de progresividad tributaria y redistribución para las hipótesis simuladas.

En tercer lugar, para arrojar más luz sobre los factores que influyen en la capacidad redistributiva del impuesto sobre la renta en el Ecuador, se calculan los tipos impositivos efectivos pagados por los grupos de ingreso más altos, utilizando las series de participación de los altos ingresos construidas por Cano (2015) para calcular los tipos impositivos sobre la renta realmente pagados por las personas de altos ingresos.

En cuarto lugar, conforme la literatura sobre elasticidad del ingreso imponible, se emplean distintos valores internacionales para la elasticidad compensada y no compensada (por ejemplo, 0,2 y 0,5), según la propuesta de Saez (2001), para obtener una gama de tipos impositivos óptimos para el grupo de ingresos correspondiente al 1\% más rico de la población.

Es necesario formular una advertencia. El análisis de este trabajo se basa en declaraciones de la renta individuales y, en consecuencia, no tiene en cuenta a los trabajadores que operan en el sector informal o a las personas cuyos ingresos no superan la deducción por gastos personales estándar (9.210 dólares en 2011), excepto por los trabajadores del sector formal que están presentes en la base de datos aunque no ganan lo suficiente como para pagar impuestos. Los datos también resultaron afectados por problemas de evasión fiscal y remoción de impuestos. Debido a las diferencias metodológicas, es probable que los resultados de este trabajo difieran de los de otros estudios sobre el efecto redistributivo del impuesto sobre la renta de las personas físicas en el Ecuador (Roca, 2009; Hanni, Martner y Podestá, 2015), basados en datos de encuestas de hogares.

\section{Resultados}

\section{Medición de la progresividad tributaria y el efecto redistributivo del impuesto sobre la renta de las personas físicas}

En esta subsección se evalúan la progresividad y la capacidad redistributiva del impuesto sobre la renta de las personas físicas en el Ecuador en el período 2007-2011 (es decir, antes y después de la

\footnotetext{
9 Existen dos tipos diferentes de deducciones del impuesto sobre la renta en el Ecuador: i) todos los costos y las deducciones que son obligatorios por ley y ii) las deducciones del impuesto sobre la renta de las personas físicas para gastos en vivienda, educación, salud, vestimenta y alimentos permitidas en el marco de la reforma tributaria de 2008.
} 
reforma tributaria de 2008). Como se mencionó en la sección anterior sobre métodos, se calculan los índices de Kakwani y Suits para analizar la progresividad del impuesto sobre la renta de las personas físicas y el índice de Reynolds-Smolensky para medir su capacidad redistributiva ${ }^{10}$. Los resultados se presentan en el cuadro 1.

\section{Cuadro 1}

Ecuador: indicadores de progresividad y redistribución del impuesto sobre la renta de las personas físicas

\begin{tabular}{lccccc}
\hline Indicador & 2007 & 2008 & 2009 & 2010 & 2011 \\
\hline Gini antes de impuestos & 0,6006 & 0,6558 & 0,6441 & 0,6378 & 0,5938 \\
\hline Gini después de impuestos & 0,5921 & 0,6483 & 0,6377 & 0,6307 & 0,5844 \\
\hline Tipo impositivo medio & 0,0249 & 0,0267 & 0,0218 & 0,0234 & 0,0291 \\
\hline Índice de Reynolds-Smolensky & 0,0085 & 0,0075 & 0,0064 & 0,0071 & 0,0093 \\
\hline Índice de progresividad de Kakwani & 0,3388 & 0,2756 & 0,2915 & 0,3023 & 0,3145 \\
\hline Índice de progresividad de Suits & 0,5711 & 0,4503 & 0,4752 & 0,528 & 0,4623 \\
\hline
\end{tabular}

Fuente: Elaboración propia, sobre la base de datos de declaraciones de la renta individuales del Servicio de Rentas Internas (SRI) del Ecuador.

El índice de progresividad de Kakwani $K$ se calcula como la diferencia entre el coeficiente de concentración de los impuestos ${ }^{11}$ y el coeficiente de Gini de los ingresos antes de impuestos. Si $K>0$, los impuestos sobre la renta son progresivos, mientras que si $K<0$ los impuestos sobre la renta son regresivos y no contribuyen a la reducción de la desigualdad de los ingresos. Los resultados del índice de Kakwani $K$ de 2007 (antes de la reforma tributaria) y de 2008 en adelante (después de la reforma tributaria), presentados en el cuadro 1, sugieren que el impuesto sobre la renta de las personas físicas es progresivo en el Ecuador, pues son positivos en todo el período estudiado: 0,34 en 2007, 0,28 en 2008, 0,29 en 2009, 0,30 en 2010 y 0,32 en 2011. Esto muestra que el porcentaje acumulativo de impuestos sobre la renta pagados por los contribuyentes más ricos es mayor que el porcentaje acumulativo de impuestos sobre la renta pagados por las personas más pobres.

El índice de Suits, interpretado en forma análoga al coeficiente de Gini, revela el mismo patrón. Por ejemplo, el valor 1 del índice de Suits corresponde a la situación hipotética en que solo las personas de altos ingresos pagan la totalidad de los impuestos sobre la renta (progresividad extrema), mientras que el valor - 1 del índice de Suits corresponde a la situación extrema en que solo las personas más pobres pagan la totalidad de los impuestos sobre la renta (regresividad extrema). Los resultados detallados en el cuadro 1 muestran que el índice de Suits es positivo en el período 2007-2011 (0,57 en 2007, 0,48 en 2009 y 0,46 en 2011), de manera que el impuesto sobre la renta de las personas físicas en el Ecuador es progresivo. Además, de acuerdo con Roca (2009) y Hanni, Martner y Podestá (2015), se calcula la proporción de los impuestos sobre la renta totales pagados por cada decil de ingresos. Al igual que esos autores, se encuentra que casi el $90 \%$ del impuesto sobre la renta de las personas físicas en el Ecuador es pagado por el decil más rico. Este resultado muestra que el impuesto sobre la renta de las personas físicas en el país es muy progresivo ${ }^{12}$.

\footnotetext{
${ }^{10}$ Los índices de progresividad y redistribución se calculan utilizando el módulo PROGRES desarrollado para Stata por Philippe Van Kerm y Andreas Peichl del Luxembourg Institute of Socio-Economic Research (LISER) y el Centre for European Economic Research (Van Kerm y Peichl, 2007).

${ }^{11}$ Como se recordará, el coeficiente de concentración de los impuestos, también conocido como coeficiente de cuasi Gini, corresponde a la curva de concentración de los impuestos.

${ }^{12}$ Este resultado probablemente refleja el hecho de que la mayoría de los contribuyentes presentes en la base de datos de declaraciones fiscales, especialmente los trabajadores, ganan menos del umbral a partir del cual se recauda el impuesto sobre la renta.
} 
Como señala Roca (2009, págs. 53-55), la medición de la progresividad y la capacidad redistributiva de los impuestos sobre la renta en los países latinoamericanos debería considerarse como un ejercicio teórico, debido a los altos niveles de evasión fiscal y remoción de impuestos y también debido a exenciones tributarias ${ }^{13}$. Desafortunadamente, los países latinoamericanos se caracterizan por grandes desigualdades de ingresos. De hecho, Hanni, Martner y Podestá (2015) muestran que la participación en el ingreso total correspondiente al decil más rico en los países latinoamericanos alcanzó una media del 32\% en 2012. Probablemente, debido a los altos niveles de concentración del ingreso, evasión fiscal y remoción de impuestos, el impuesto sobre la renta de las personas físicas en el Ecuador es en realidad menos progresivo de lo que se documenta en esta sección. Por ese motivo, y para arrojar nueva luz sobre la progresividad de los impuestos sobre la renta, en la sección III.3 se calculan los tipos impositivos efectivos pagados por los grupos de ingresos más altos.

En síntesis, los resultados de los índices de Kakwani y Suits y de la carga del impuesto sobre la renta de las personas físicas sugieren que, en líneas generales, el impuesto sobre la renta de las personas físicas en el Ecuador es progresivo. Estos resultados concuerdan con las tendencias halladas por Roca (2009) y Hanni, Martner y Podestá (2015), que se basan en encuestas de hogares.

Desde un punto de vista teórico, los impuestos sobre la renta progresivos "empujan" la curva de Lorenz de los ingresos antes de impuestos hacia la diagonal, reduciendo la desigualdad de la distribución del ingreso después de impuestos. La magnitud de este movimiento se capta mediante el índice de Reynolds-Smolensky, que mide la diferencia entre el coeficiente de Gini de los ingresos antes de impuestos y el coeficiente de Gini de los ingresos después de impuestos. Los resultados del índice de Reynolds-Smolensky para el Ecuador también se muestran en el cuadro 1. A primera vista, puede observarse que el coeficiente de Gini antes de impuestos es mayor que el coeficiente de Gini después de impuestos a lo largo del período estudiado, lo que sugiere que el impuesto sobre la renta de las personas físicas efectivamente reduce la desigualdad de los ingresos. Sin embargo, esta reducción es tan pequeña que resulta casi inobservable, de manera que no hay cambios significativos en el índice de Reynolds-Smolensky en ninguno de los años examinados. El coeficiente de Gini después de impuestos sobre la renta disminuye de 0,655 a 0,648 en 2008 y de 0,637 a 0,630 en 2010. Estos resultados indican que si bien el impuesto sobre la renta de las personas físicas en el Ecuador es progresivo, como muestran los índices de Kakwani y Suits, su capacidad redistributiva es muy débil. Además, no se observan cambios significativos entre 2007 y 2008, es decir antes y después de la reforma tributaria, que se promulgó precisamente para incrementar la progresividad tributaria y, en consecuencia, la redistribución del ingreso.

Para arrojar nueva luz sobre los factores que erosionan la capacidad redistributiva del impuesto sobre la renta de las personas físicas, se estudian las nuevas deducciones del impuesto sobre la renta de las personas físicas introducidas por la reforma tributaria de 2008 en el período 2008-201014. En los gráficos 1 y 2 se representa la curva de Lorenz de los ingresos antes de impuestos en 2008 y 2010, respectivamente (líneas de color rojo oscuro), junto con las curvas de concentración para cada tipo de deducción del impuesto sobre la renta de las personas físicas y para las obligaciones tributarias del impuesto sobre la renta ${ }^{15}$.

\footnotetext{
${ }^{13}$ Roca (2009) sugiere que casi el 70\% de las personas con menores ingresos en el Ecuador no ganan lo suficiente para pagar impuestos y, en consecuencia, la evasión fiscal concierne principalmente a las personas de altos ingresos.

${ }^{14}$ Desafortunadamente, no se tuvo acceso a los microdatos sobre las deducciones del impuesto sobre la renta de las personas físicas correspondientes a 2011.

${ }^{15}$ Las curvas de concentración representan el porcentaje acumulativo de diferentes deducciones del impuesto sobre la renta de las personas físicas y obligaciones tributarias en relación con el porcentaje acumulativo de la población clasificada por ingresos totales $\left(C_{p}\right)$.
} 


\section{Gráfico 1}

Ecuador: curvas de Lorenz y de concentración para las deducciones del impuesto sobre la renta y las obligaciones tributarias, 2008

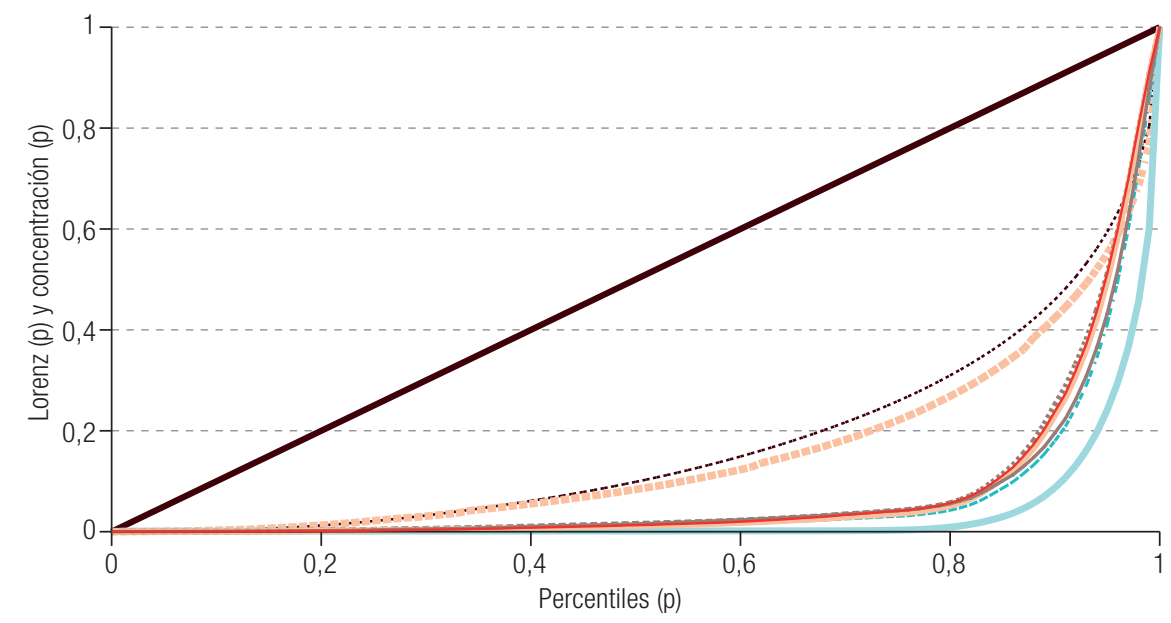

- Línea de $45^{\circ}$

- 1 - Concentración (p): deducción de costos

...... Lorenz (p): ingresos de 2008

— Concentración (p): deducción fiscal para vestimenta

_ Concentración (p): impuesto sobre la renta

-- - - Concentración (p): deducción fiscal para educación

- "u. Concentración ( $p)$ : deducción fiscal para alimentos

— Concentración (p): deducción fiscal para vivienda

- Concentración (p): deducción fiscal para salud

Fuente: Elaboración propia, sobre la base de datos de declaraciones de la renta individuales del Servicio de Rentas Internas (SRI) del Ecuador.

Nota: En el gráfico se representa la curva de Lorenz de los ingresos brutos antes de impuestos junto con diferentes curvas de concentración para las deducciones del impuesto sobre la renta y las obligaciones tributarias en 2008.

\section{Gráfico 2}

Ecuador: curvas de Lorenz y de concentración para las deducciones del impuesto sobre la renta y las obligaciones tributarias, 2010

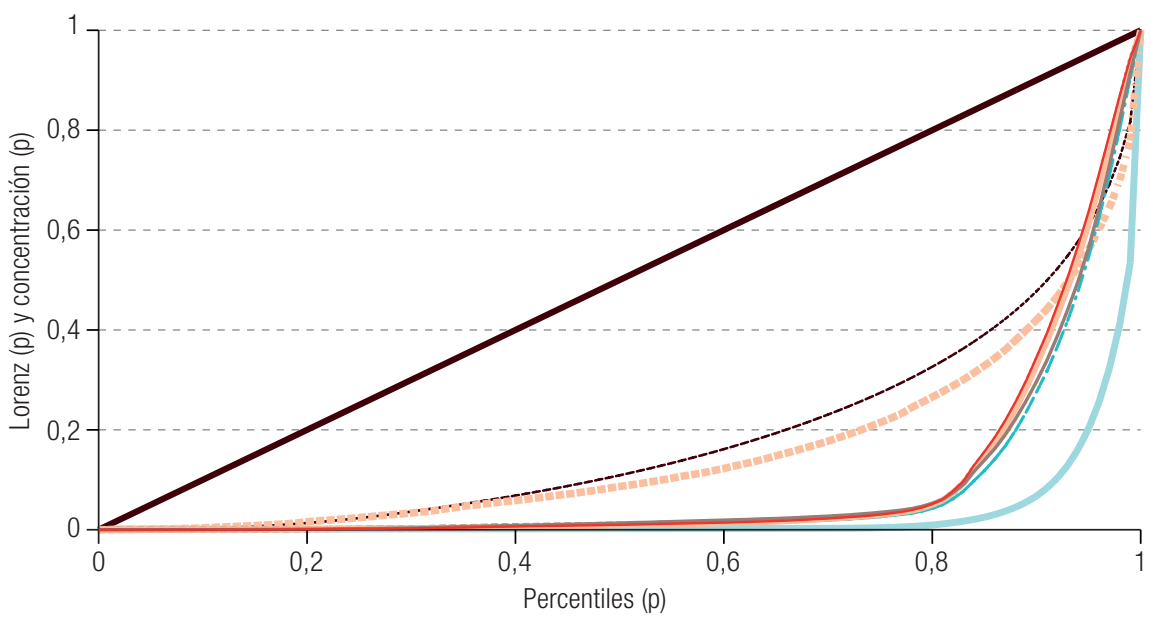

\footnotetext{
- Línea de $45^{\circ}$

- Concentración (p): impuesto sobre la renta

— Concentración (p): deducción fiscal para vestimenta

-- - - Concentración (p): deducción fiscal para educación

— Concentración (p): deducción fiscal para vivienda
}

...... Lorenz (p): ingresos de 2010

m * - Concentración (p): deducción de costos

..... Concentración (p): deducción fiscal para alimentos

— Concentración (p): deducción fiscal para salud

Fuente: Elaboración propia, sobre la base de datos de declaraciones de la renta individuales del Servicio de Rentas Internas (SRI) del Ecuador

Nota: En el gráfico se representa la curva de Lorenz de los ingresos brutos antes de impuestos junto con diferentes curvas de concentración para las deducciones del impuesto sobre la renta y las obligaciones tributarias en 2010. 
De los gráficos 1 y 2 surgen varias observaciones. En primer lugar, la curva de concentración de los impuestos (trazada en verde) se encuentra por debajo de la curva de Lorenz antes de impuestos en ambos gráficos e indica que las obligaciones tributarias están distribuidas de manera más desigual que el ingreso ${ }^{16}$. Este resultado concuerda con la evidencia previa de los índices de Kakwani y Suits, que indicaban que el impuesto sobre la renta de las personas físicas era progresivo.

En segundo lugar, los gráficos 1 y 2 muestran claramente que los contribuyentes que más se beneficiaron de las deducciones del impuesto sobre la renta de las personas físicas son aquellos en los percentiles octogésimo y superiores. Al interpretar de manera similar las curvas de concentración de las obligaciones tributarias, se observa que la proporción de deducciones del impuesto sobre la renta de las personas físicas de los contribuyentes de altos ingresos es mayor que la de los contribuyentes más pobres. En otras palabras, las personas de altos ingresos (del octogésimo percentil hacia arriba) son las beneficiarias de la mayoría de las nuevas deducciones del impuesto sobre la renta de las personas físicas introducidas por la reforma tributaria de 2008. Cano (2015) muestra que la participación en el ingreso del 5\% más rico de la población en el Ecuador, controlada por variables de ingresos totales y población total, representó los 22 centiles más altos de la distribución del ingreso tributario en 2008 y los 19 centiles más altos en 2011 (sin la variable de control de la población total). Así, los gráficos 1 y 2 muestran que el $5 \%$ más rico fue el que más se benefició de las deducciones del impuesto sobre la renta de las personas físicas.

Si bien todos los indicadores sugieren que el diseño del impuesto sobre la renta de las personas físicas en el Ecuador es en general progresivo, un análisis detallado de las deducciones del impuesto sobre la renta muestra que las personas de altos ingresos tienen más probabilidades de reducir su ingreso imponible mediante deducciones del impuesto sobre la renta de las personas físicas que las personas de medios y bajos ingresos, erosionando el efecto redistributivo de la tributación progresiva. Este resultado concuerda con los del índice de Reynolds-Smolensky presentados en el cuadro 1, que llevan a confirmar que la capacidad redistributiva del impuesto sobre la renta de las personas físicas en el Ecuador - como en la mayoría de los países latinoamericanos - es débil (Roca, 2009). Las simulaciones realizadas por la CEPAL también subrayaron que el gasto público dirigido utilizando ingresos generados por un impuesto sobre la renta de las personas físicas más fuerte es en general más redistributivo que las mejoras asociadas con dicho impuesto en sí.

\section{Microsimulación e incidencia del impuesto sobre la renta: hipótesis alternativas}

Para analizar con mayor profundidad el efecto redistributivo del impuesto sobre la renta de las personas físicas, en esta sección se emplean técnicas de microsimulación a nivel individual para captar los cambios en la distribución del ingreso antes de impuestos cuando se reducen o eliminan las deducciones del impuesto sobre la renta de las personas físicas impuestas por ley. Sobre la base de la sección precedente, en la que se demostró empíricamente que los más beneficiados por las deducciones del impuesto sobre la renta de las personas físicas eran los contribuyentes que pertenecen al 5\% más rico de la población ${ }^{17}$ (cuyo ingreso imponible es el que más se reduce por las deducciones fiscales), el principal objetivo de esta subsección consiste en determinar si el efecto redistributivo del impuesto sobre la renta de las personas físicas aumenta cuando se modifican las deducciones fiscales.

\footnotetext{
${ }^{16}$ En otras palabras, la proporción de obligaciones tributarias de los contribuyentes de bajos ingresos es menor que la de los contribuyentes ricos.

${ }^{17}$ Como se recordará, los totales de ingresos y de población se utilizan como controles externos para construir los grupos de participación de mayores ingresos.
} 
Los modelos de microsimulación se caracterizan a menudo como estáticos, dinámicos y conductuales ${ }^{18}$. Los modelos de microsimulación estáticos se emplean tradicionalmente para evaluar el efecto inmediato de las modificaciones de política en las personas. En estos, las microunidades de observación se mantienen constantes y no se tienen en cuenta los cambios en curso en la composición demográfica o los cambios conductuales debidos a variaciones de política o a la dimensión temporal. Por el contrario, los modelos de microsimulación dinámicos contienen parámetros específicos que estiman las respuestas de las personas a los cambios de política y se emplean generalmente para analizar resultados a largo plazo, efectos intergeneracionales y proyecciones socioeconómicas y demográficas en el marco de las políticas actuales, entre otras cosas (Bourguignon y Spadaro, 2006). Los modelos conductuales, por otra parte, utilizan herramientas microeconométricas para estimar los efectos de las modificaciones de política en la conducta de las personas, generalmente con respecto a la oferta de trabajo (Figari, Paulus y Sutherland, 2015).

Se emplea un modelo de microsimulación estático para analizar la distribución del ingreso antes y después de impuestos y las obligaciones tributarias en el marco de diferentes hipótesis simuladas. El principal objetivo es proporcionar una idea general de los posibles efectos redistributivos de la modificación de las deducciones del impuesto sobre la renta de las personas físicas. Debido a que el enfoque de microsimulación adoptado es estático, no se incorporan cambios en la conducta de los individuos como resultado de las modificaciones de los impuestos. A partir de los datos a nivel micro, por cada contribuyente se simula aritméticamente un ingreso antes de impuestos para dos hipótesis contrarias a los hechos ${ }^{19}$. En la primera hipótesis se prevé un $50 \%$ de costos y deducciones y se eliminan las deducciones del impuesto sobre la renta de las personas físicas. En la segunda se eliminan todas las deducciones del impuesto sobre la renta. A continuación se emplean los tipos impositivos y cuadros de impuestos legales para 2008, 2009 y 2010 para simular las nuevas obligaciones tributarias y los ingresos después de impuestos para cada persona en el marco de ambas hipótesis.

Las hipótesis base y simuladas se construyen de la siguiente manera:

Hipótesis base 0: $Y_{0}-t_{0}$, donde $Y_{0}$ son los ingresos brutos y $t_{0}$ son las obligaciones tributarias declaradas por los contribuyentes.

Hipótesis 1: $Y_{0}-t_{1}$, donde $Y_{0}$ son los ingresos brutos y $t_{1}$ son las obligaciones tributarias simuladas cuando se prevé un $50 \%$ de costos y deducciones y se eliminan todas las deducciones del impuestos sobre la renta de las personas físicas.

Hipótesis 2: $Y_{0}-t_{2}$, donde $Y_{0}$ son los ingresos brutos y $t_{2}$ son las obligaciones tributarias simuladas cuando se eliminan todas las deducciones fiscales.

A continuación se utiliza la metodología descrita en la subsección 1 para calcular los indicadores de desigualdad, progresividad y redistribución de las hipótesis simuladas.

En el cuadro 2 se presentan los resultados de la evaluación de la política tributaria en un período de tres años. En 2008, los índices de progresividad de Kakwani y Suits son positivos en todas las hipótesis simuladas, de manera que el impuesto sobre la renta de las personas físicas se mantiene progresivo, al igual que en la hipótesis base. Este resultado era de esperarse, pues no se crean nuevos tipos impositivos marginales ni nuevas categorías de impuesto sobre la renta en las hipótesis 1 y 2 y en las simulaciones de hecho se emplean los mismos tipos impositivos que en la hipótesis 0. Sin embargo, el índice de Reynolds-Smolensky, que capta la diferencia en el coeficiente de Gini antes y después del impuesto sobre la renta de las personas físicas, cambia más sustancialmente en las dos hipótesis simuladas.

\footnotetext{
${ }^{18}$ Spadaro (2007) y Figari, Paulus y Sutherland (2015) ofrecen un excelente resumen de los enfoques de microsimulación y diferentes modelos de microsimulación tradicionalmente empleados para evaluar los efectos de las políticas públicas.

19 Una hipótesis contraria a los hechos muestra lo que ocurriría si se implementara un cambio de política.
} 


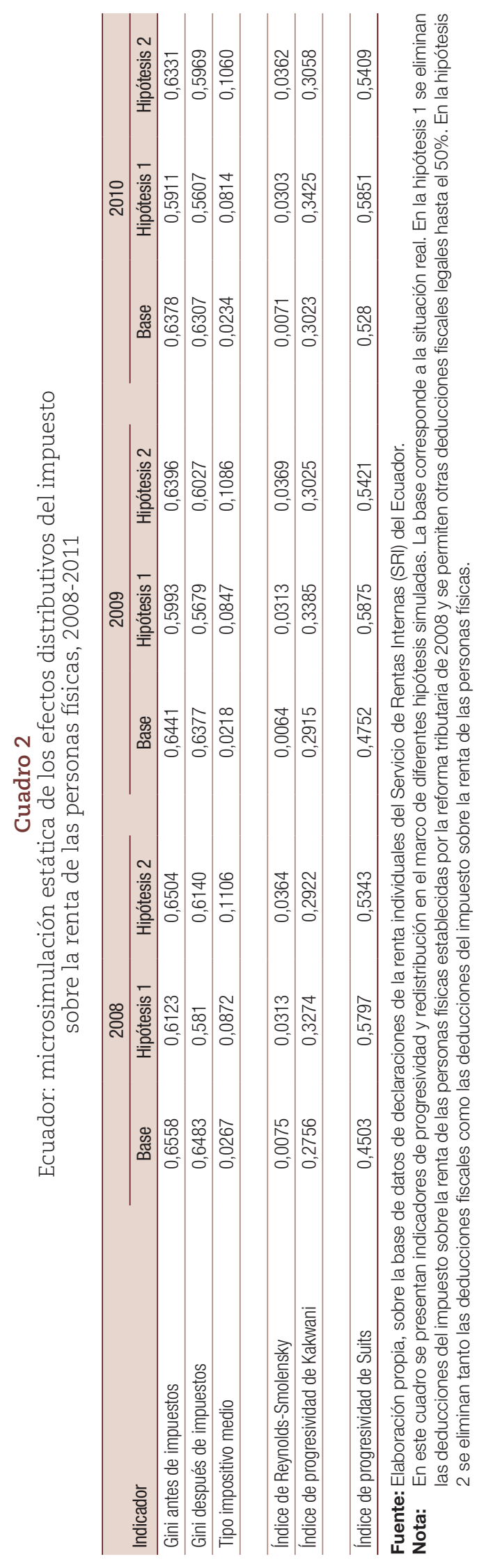


Si bien el efecto redistributivo del impuesto sobre la renta de las personas físicas es muy débil en la hipótesis 0 (base), en la que el coeficiente de Gini disminuye de 0,656 antes de impuestos a 0,648 después de impuestos, en la hipótesis simulada 1, en la que se eliminan las nuevas deducciones del impuesto sobre la renta de las personas físicas, presenta un efecto redistributivo mayor.

De hecho, el índice de Reynolds-Smolensky aumenta de 0,0075 (base) a 0,0313 (hipótesis 1) y la desigualdad de los ingresos, medida por el coeficiente de Gini, disminuiría casi 3 puntos, de 0,61 a 0,58 , después de la política tributaria. En otras palabras, los resultados simulados de las hipótesis 1 y 2 sugieren que el efecto redistributivo del impuesto sobre la renta de las personas físicas sería mayor si no se hubieran introducido deducciones de dicho impuesto mediante la reforma tributaria de 2008. El mismo patrón se observa con respecto a 2009 y 2010. En el cuadro 2 se muestra que el impuesto sobre la renta de las personas físicas es progresivo en todas las hipótesis simuladas. Además, al eliminar las deducciones del impuesto sobre la renta de las personas físicas (hipótesis simulada 1), el efecto redistributivo de la tributación directa aumenta.

Los resultados de la hipótesis 2, en la que se eliminan todas las deducciones fiscales (deducciones del impuesto sobre la renta de las personas físicas y otras deducciones legales), son bastante similares a los de la hipótesis 1 en todos los años. El efecto redistributivo del impuesto sobre la renta de las personas físicas, medido por el índice de Reynolds-Smolensky, aumenta casi 4 puntos y el coeficiente de Gini disminuye de 0,65 antes del impuesto sobre la renta a 0,61 después de impuestos en 2008. En 2009 y 2010 se observan patrones similares.

En resumen, los resultados de las hipótesis simuladas confirman que el sistema del impuesto sobre la renta de las personas físicas es progresivo. Asimismo, esas hipótesis muestran que el efecto redistributivo de los impuestos sobre la renta podría ser mayor si se regularan las deducciones fiscales en general. Si bien se simularon casos extremos en que se eliminan todas las deducciones fiscales, es sabido que algunos tipos de deducciones son necesarios para aumentar la equidad del impuesto sobre la renta. En el Ecuador, la introducción de deducciones del impuesto sobre la renta de las personas físicas ha creado incentivos para que las personas, especialmente las que pertenecen al $5 \%$ más rico de la población, reduzcan su ingreso imponible y, en consecuencia, sus obligaciones tributarias. Como se sugirió anteriormente, esto anula el efecto de progresividad teóricamente buscado por la reforma tributaria. Por lo tanto, es imprescindible regular adecuadamente las exenciones y las deducciones fiscales en el país.

Para examinar otros factores que erosionan el efecto redistributivo del impuesto sobre la renta de las personas físicas, en la próxima sección se analizan los tipos impositivos efectivos pagados por los individuos de altos ingresos en el período 2007-2011. Sobre la base de la literatura en materia de elasticidad del ingreso imponible se calculan tipos impositivos óptimos para los grupos de altos ingresos y se formulan recomendaciones de política.

\section{Tipos impositivos efectivos pagados por los grupos de ingresos más altos}

\section{a) Construcción de series de participación de los altos ingresos en el ingreso total}

El análisis comienza con la construcción de series de participación de los altos ingresos en el ingreso total en el período 2007-2011. Al igual que en Cano (2015), se sigue la literatura estándar sobre altos ingresos (Piketty, 2001; Piketty y Saez, 2003; Atkinson y Piketty, 2007; Atkinson, Piketty y Saez, 2011) y se construyen la serie del grupo de ingresos correspondiente al 1\% más rico de la 
población (denominada P99-100) y las series para un conjunto de fractiles más pequeños: P99.5-100 (0,5\%), P99.9-100 (0,1\%) y P99.99-100 (0,01\%).

Cada fractil se construye con respecto al número total de potenciales contribuyentes en toda la población ecuatoriana de adultos de 20 o más años de edad, que, como señala Piketty (2003), no debe confundirse con el número real de declaraciones fiscales presentadas. El ingreso se define como previo al impuesto sobre la renta de las personas físicas. Esta definición incluye todos los ítems declarados en las declaraciones fiscales: sueldos y salarios, pensiones, ingresos por trabajo autónomo, ingresos netos de empresas no constituidas en sociedades de capital, dividendos, intereses, otros ingresos de capital y alquiler y otros ítems de ingresos. A continuación se calculan las participaciones en el ingreso dividiendo los montos de ingresos que corresponden a cada fractil (P99-100, P99.9100, P99.99-100) por un total de ingresos de control. Atkinson, Piketty y Saez (2011) proponen dos métodos distintos para calcular este control. El primero se basa en los datos del impuesto sobre la renta y suma los ingresos de los no declarantes, mientras que el segundo calcula un total de control externo derivado de las cuentas nacionales. En este trabajo, como en Cano (2015), se adopta el segundo enfoque, también empleado por Piketty (2001) y Piketty y Saez (2003), y se construye un total de control para los ingresos a partir de las cuentas nacionales de la siguiente manera: saldo de ingresos primarios de los hogares más prestaciones sociales distintas de las transferencias sociales en especie, menos contribuciones sociales efectivas de los empleadores, menos contribuciones sociales imputadas de los empleadores, menos ingreso de la propiedad atribuido a titulares de póliza de seguros, menos alquiler imputado por propiedad ocupada por el dueño, menos consumo de capital fijo. Este enfoque produce una variable de control para el ingreso total del $59 \%$ al $66 \%$ del producto interno bruto (PIB), según el año. Los resultados muestran que la proporción del ingreso total del 1\% más rico de la población en 2010 varía del 14\% al 17\%, según la definición del ingreso empleada como numerador de la proporción ${ }^{20}$.

Una vez construidas las series de participación de los altos ingresos, se analizan los tipos impositivos efectivos pagados por esos grupos ${ }^{21}$. Este artículo constituye un aporte a la creciente literatura en la que se analiza no solo la dinámica de los altos ingresos sino también sus repercusiones en materia de política tributaria (Piketty y Saez, 2003; Saez y Veall, 2005; Landais, Piketty y Saez, 2011; Alvaredo y Londoño, 2013; Burdín, Esponda y Vigorito, 2014; Fairfield y Jorratt, 2016).

En el cuadro 3 se presentan los resultados de los tipos impositivos efectivos pagados por los grupos de ingresos más altos. Si bien el ingreso de las personas físicas en el Ecuador está gravado a tipos marginales progresivos que van del $0 \%$ al 35\%, los tipos impositivos efectivos pagados por las personas de muy altos ingresos son menores debido a deducciones fiscales, exenciones tributarias y probablemente a la evasión fiscal. En el período 2007-2011, el grupo de ingresos correspondiente al $1 \%$ más rico de la población pagó un tipo impositivo efectivo medio de alrededor del $7 \%$ y el 0,1\% más rico un tipo impositivo efectivo medio del 9,4\%. Los resultados muestran que, excepto en 2010, los tipos impositivos efectivos medios disminuyeron dentro de los grupos de mayores ingresos, especialmente entre los grupos correspondientes al 0,1\%, el 0,05\%, el 0,01\% y el 0,001\% más ricos. Por ejemplo, mientras que el tipo impositivo efectivo medio del $1 \%$ más rico de la población fue de casi el 6,3\% en 2009, el tipo impositivo medio del 0,001\% más rico fue del 1,9\%.

Esta situación se relaciona con la composición del ingreso en los grupos en la cima de la distribución y con el hecho de que el ingreso de capital está menos gravado que el ingreso correspondiente al salario. Cano (2015) analizó la composición del ingreso en los grupos de mayores

\footnotetext{
${ }^{20}$ Para ofrecer un panorama preciso de los ingresos en la cima de la distribución, Cano (2015) construyó series de participación de los altos ingresos en el ingreso total en el Ecuador con diferentes definiciones de ingreso como numerador de la proporción.

${ }^{21}$ Los tipos impositivos efectivos se definen como los impuestos sobre la renta individuales pagados divididos por los ingresos por trabajo declarados.
} 
ingresos y encontró que en 2011 el ingreso del 1\% más rico estaba compuesto principalmente por sueldos (45\%) e ingresos de actividades empresariales (27\%) o trabajo autónomo (21\%) y solo en menor medida por ingreso de capital (7\%). Mientras tanto, el ingreso del 0,01\% más rico comprendía una menor proporción de sueldos (16\%) pero mayores proporciones de ingresos provenientes de actividades empresariales (29\%), trabajo autónomo (28\%) y capital (27\%). Cabe subrayar cuánto ha variado la composición del ingreso en el decil más alto (P90-100). Mientras que la proporción del ingreso por sueldos claramente disminuyó en la parte superior de la escala, aquella del empleo autónomo y las actividades empresariales aumentó hasta el 0,1\%-0,01\% más rico antes de volver a disminuir hacia el grupo correspondiente al 0,01\%. Por el contrario, la proporción de ingreso de capital aumentó en la cima de la distribución. Por ejemplo, mientras la proporción de capital era del $4 \%$ entre el $10 \%$ más rico y apenas por debajo del 1,7\% en el grupo correspondiente al 10\%-5\% más rico en 2011, esta alcanzaba el 50\% entre el 0,001\% con mayores ingresos. La creciente proporción de ingresos de capital en los grupos de muy altos ingresos sugiere que aquellos cuyos ingresos provienen de esta fuente constituyen el grupo más grande en la cima de la distribución del ingreso en el Ecuador.

El cuadro 3 revela una marcada diferencia entre la situación en 2007 y aquella a partir de 2008, pues los tipos impositivos medios para los grupos de muy altos ingresos disminuyeron con respecto a su nivel de 2007, aunque se recuperaron considerablemente en 2010. La disminución entre 2007 y 2009 probablemente se explica porque las personas de altos ingresos redujeron su ingreso imponible mediante deducciones fiscales, en particular las nuevas deducciones sobre la renta de las personas físicas permitidas por la ley tributaria de 2008. En esta subsección se mostró gráficamente que las nuevas deducciones sobre la renta de las personas físicas se concentraron en gran medida en el extremo superior de la distribución (es decir, del octogésimo percentil en adelante o el 5\% más rico cuando se utiliza un total de control para la población). Los resultados presentados en esta sección sugieren que el $0,1 \%$ más rico es probablemente el que más se ha beneficiado de este tipo de deducción fiscal.

\section{Cuadro 3}

Ecuador: tipos impositivos efectivos medios sobre la renta en los grupos de ingresos más altos, 2007-2011

\begin{tabular}{rrrrrrrrrrrrrr}
\hline Año & $\begin{array}{c}10 \% \\
\text { más } \\
\text { rico }\end{array}$ & $\begin{array}{c}\text { más } \\
\text { rico }\end{array}$ & $\begin{array}{c}1 \% \\
\text { más } \\
\text { rico }\end{array}$ & $\begin{array}{c}0,5 \% \\
\text { más } \\
\text { rico }\end{array}$ & $\begin{array}{c}0,1 \% \\
\text { más } \\
\text { rico }\end{array}$ & $\begin{array}{c}0,05 \% \\
\text { más } \\
\text { rico }\end{array}$ & $\begin{array}{c}0,01 \% \\
\text { más } \\
\text { rico }\end{array}$ & $\begin{array}{c}0,001 \% \\
\text { más } \\
\text { rico }\end{array}$ & $\begin{array}{c}1,0 \%- \\
0,5 \% \\
\text { más } \\
\text { rico }\end{array}$ & $\begin{array}{c}0,5 \%- \\
0,1 \% \\
\text { más } \\
\text { rico }\end{array}$ & $\begin{array}{c}0,1 \%- \\
0,05 \% \\
\text { más } \\
\text { rico }\end{array}$ & $\begin{array}{c}0,05 \%- \\
0,01 \% \\
\text { más } \\
\text { rico }\end{array}$ & $\begin{array}{c}0,01 \%- \\
0,001 \% \\
\text { más } \\
\text { rico }\end{array}$ \\
\hline 2007 & 3,1 & 4,0 & 7,4 & 9,0 & 12,4 & 13,3 & 12,2 & 6,8 & 3,6 & 6,4 & 10,3 & 14,2 & 15,1 \\
\hline 2008 & 3,7 & 4,7 & 7,2 & 8,0 & 8,5 & 7,7 & 5,8 & 2,4 & 5,1 & 7,5 & 10,3 & 9,4 & 7,6 \\
\hline 2009 & 3,1 & 3,9 & 6,3 & 6,9 & 7,0 & 6,2 & 4,4 & 1,9 & 4,4 & 6,9 & 9,1 & 8,0 & 5,9 \\
\hline 2010 & 3,3 & 4,2 & 7,0 & 7,9 & 9,5 & 9,7 & 9,6 & 8,8 & 4,3 & 6,5 & 8,9 & 9,9 & 9,9 \\
\hline 2011 & 4,7 & 6,2 & 8,7 & 9,3 & 9,7 & 9,2 & 6,6 & 3,7 & 7,3 & 9,0 & 10,9 & 10,7 & 8,9 \\
\hline
\end{tabular}

Fuente: Elaboración propia, sobre la base de datos de declaraciones de la renta individuales del Servicio de Rentas Internas (SRI) del Ecuador.

Nota: En este cuadro se muestran los tipos impositivos efectivos medios en los grupos de ingresos más altos, utilizando el criterio de ingresos brutos (ingresos empresariales netos de costos y deducciones).

Estos resultados llevan a creer que a pesar del alto nivel de concentración del ingreso, las personas de altos ingresos pagan tipos impositivos sobre la renta efectivos muy bajos. Además, aunque todos los indicadores sintéticos (que son más sensibles a los cambios en el medio de la distribución que en las colas) sugieren que el impuesto sobre la renta de las personas físicas en el Ecuador es progresivo, un análisis más detallado de los tipos impositivos realmente pagados por las personas de altos ingresos muestra que estos disminuyen entre los grupos de ingresos más altos. 


\section{Uso de elasticidades para obtener tipos impositivos óptimos para las personas de altos ingresos}

Esta subsección se basa en la literatura sobre elasticidad del ingreso imponible para obtener tipos impositivos óptimos para los grupos de ingresos más altos. Se emplean diferentes valores de elasticidades compensadas y no compensadas conforme la propuesta de Saez (2001) y se obtiene una gama de tipos impositivos óptimos para el grupo de ingreso correspondiente al 1\% más rico de la población. Desafortunadamente, no se pudo calcular la elasticidad del ingreso imponible con respecto a los tipos marginales utilizando la reforma tributaria de 2008 como un experimento natural, porque los resultados estaban muy influenciados por cambios en la cobertura de la población entre 2007 y 2008. El porcentaje de adultos incluidos en la base de datos de declaraciones fiscales aumentó del 18\% en 2007 a casi el 27\% en 2011 (véase el gráfico en el anexo A1). Debido a que los cambios en la cobertura de la población distorsionaron los resultados de elasticidad, se decidió emplear valores de elasticidad estándar propuestos en la literatura. Fairfield (2010) procedió de la misma manera para estimar tipos impositivos óptimos para las personas de altos ingresos en Chile y la Argentina ${ }^{22}$.

Se procede de la siguiente manera. En primer lugar, conforme la literatura sobre altos ingresos (Atkinson, Piketty y Saez, 2011), se calcula el coeficiente de Pareto invertido $(\beta)$ para el período 2007-2011 de la siguiente manera:

$$
\begin{gathered}
\alpha=\frac{1}{1-\left[\log \frac{S_{1}}{S_{0.1}} / \log 10\right]} \\
\beta=\frac{\alpha}{\alpha-1}
\end{gathered}
$$

donde $S_{1}$ es la participación en el ingreso del $1 \%$ más rico y $S_{0.1}$ la participación en el ingreso del 0,1\% más rico. Como señalan Atkinson, Piketty y Saez (2011), un coeficiente de Pareto invertido $\beta$ más alto produce una cola superior más plana en la distribución. Según la explicación propuesta por esos autores, si $\beta=2$ entonces el ingreso medio de las personas con ingresos superiores a 100.000 dólares es 200.000 dólares y el ingreso medio de las personas con ingresos superiores a 1 millón de dólares es 2 millones de dólares. Una vez construidos los parámetros de Pareto $\beta$, se sigue la propuesta de Saez (2001) y se obtienen tipos impositivos óptimos mediante la aplicación de su fórmula de la manera siguiente:

$$
\tau=\frac{1-g}{1-g+\zeta^{u}+\zeta^{c}(a-1)}
$$

donde $\tau$ es el tipo impositivo óptimo, $g$ el objetivo redistributivo del gobierno ${ }^{23}, a-1$ el parámetro de Pareto, $\xi^{u}$ la elasticidad no compensada y $\zeta^{c}$ la elasticidad compensada. Como sugiere Saez, el caso $g=0$ corresponde a la situación en que el gobierno no valora el consumo marginal de las

\footnotetext{
${ }^{22}$ Se calculó la elasticidad del ingreso imponible con respecto a los tipos impositivos marginales para las personas de altos ingresos conforme Riihela, Sullstrom y Tuomala (2014), es decir: $\epsilon=\frac{\left(\log S_{1}-\log S_{0}\right)}{\log \left(1-t_{1}\right)-\log \left(1-t_{0}\right)}$, donde $S_{1}$ es la participación en el ingreso del $1 \%$ más rico después de la reforma tributaria, $S_{0}$ corresponde al $1 \%$ más rico antes de la reforma, $t_{1}$ el tipo impositivo marginal máximo después de la reforma y $t_{0}$ el tipo impositivo marginal antes de la reforma. Desafortunadamente, los resultados estuvieron muy influenciados por el aumento en la cobertura de la población adulta entre 2007 y 2008.

${ }^{23}$ El concepto de una función de bienestar social en el cálculo del tipo impositivo marginal máximo está bien documentado en Saez (2001) y Diamond y Saez (2011).
} 
personas de altos ingresos y establece el mayor tipo máximo posible para maximizar su recaudación tributaria de estos. La fórmula propuesta por Saez está especializada sobre todo para $g=0$. Además, como sugiere Saez, las estimaciones de tipos impositivos óptimos aquí realizadas se ajustan por la presencia de un impuesto sobre el valor agregado (IVA) del 12\% de la siguiente manera: $(1-t)^{\star}$, donde $\tau$ es el tipo impositivo al consumo.

Debido a que no hay consenso en la literatura sobre elasticidad del ingreso imponible con respecto a valores fijos para la elasticidad del ingreso imponible de las personas de altos ingresos, se procede como Saez (2001) y Fairfield (2010) para obtener tipos impositivos óptimos mediante la prueba de diferentes valores de elasticidad de la siguiente manera: elasticidades no compensadas de $0,0,2$ y 0,5 y elasticidades compensadas de 0,2, 0,5 y 0,8. Además, se presentan ambos resultados cuando $g=0$ y $g=0,25$.

\section{Cuadro 4}

Ecuador: tipos impositivos óptimos para personas de altos ingresos derivados de la elasticidad, 2007-2011

\begin{tabular}{|c|c|c|c|c|c|c|c|c|c|}
\hline \multirow{2}{*}{$\begin{array}{l}\text { Elasticidad no compensad } \\
\text { Elasticidad compensada }\end{array}$} & & \multicolumn{3}{|c|}{0} & \multicolumn{3}{|c|}{0,2} & \multicolumn{2}{|c|}{0,5} \\
\hline & & 0,2 & 0,5 & 0,8 & 0,2 & 0,5 & 0,8 & 0,5 & 0,8 \\
\hline \multicolumn{10}{|l|}{$g=0$} \\
\hline Año & $\beta$ & & & & & & & & \\
\hline 2007 & 1,979 & $74 \%$ & $59 \%$ & $49 \%$ & $63 \%$ & $52 \%$ & $44 \%$ & $44 \%$ & $39 \%$ \\
\hline 2008 & 2,023 & $73 \%$ & $58 \%$ & $48 \%$ & $63 \%$ & $51 \%$ & $44 \%$ & $44 \%$ & $38 \%$ \\
\hline 2009 & 2,078 & $72 \%$ & $57 \%$ & $47 \%$ & $62 \%$ & $51 \%$ & $43 \%$ & $43 \%$ & $37 \%$ \\
\hline 2010 & 1,961 & $74 \%$ & $59 \%$ & $50 \%$ & $63 \%$ & $52 \%$ & $45 \%$ & $44 \%$ & $39 \%$ \\
\hline 2011 & 1,953 & $74 \%$ & $60 \%$ & $50 \%$ & $63 \%$ & $52 \%$ & $45 \%$ & $45 \%$ & $39 \%$ \\
\hline \multicolumn{10}{|l|}{$g=0,25$} \\
\hline Año & $\beta$ & & & & & & & & \\
\hline 2007 & 1,979 & $70 \%$ & $53 \%$ & $43 \%$ & $58 \%$ & $46 \%$ & $38 \%$ & $38 \%$ & $32 \%$ \\
\hline 2008 & 2,023 & $69 \%$ & $52 \%$ & $42 \%$ & $57 \%$ & $45 \%$ & $37 \%$ & $37 \%$ & $32 \%$ \\
\hline 2009 & 2,078 & $68 \%$ & $51 \%$ & $41 \%$ & $57 \%$ & $44 \%$ & $36 \%$ & $37 \%$ & $31 \%$ \\
\hline 2010 & 1,961 & $70 \%$ & $54 \%$ & $43 \%$ & $58 \%$ & $46 \%$ & $38 \%$ & $38 \%$ & $33 \%$ \\
\hline 2011 & 1,953 & $70 \%$ & $54 \%$ & $44 \%$ & $58 \%$ & $46 \%$ & $39 \%$ & $38 \%$ & $33 \%$ \\
\hline
\end{tabular}

Fuente: Elaboración propia, sobre la base de datos de declaraciones de la renta individuales del Servicio de Rentas Internas (SRI) del Ecuador.

Nota: Conforme Saez (2001), en este cuadro se muestran los tipos impositivos óptimos para las personas de altos ingresos. $g$ es la razón de utilidad marginal social con ingreso infinito sobre el valor marginal de los fondos públicos, $\beta$ es el parámetro de Pareto, $\xi^{U}$ es la elasticidad no compensada y $\xi^{C}$ es la elasticidad compensada. Los resultados se basan en datos de declaraciones de la renta. El parámetro de Pareto $\beta$ se calculó utilizando series de participación de los altos ingresos en el ingreso total. Los tipos impositivos óptimos se ajustan para la presencia de un IVA del 12\%.

En el cuadro 4 se muestran los tipos impositivos marginales máximos óptimos calculados para el Ecuador. Al asumir que las elasticidades son las mismas que en los Estados Unidos, casi 0,2 (tanto compensadas como no compensadas), el tipo impositivo marginal máximo óptimo para las personas de altos ingresos varía entre el 57\% y el 63\%. Los tipos impositivos marginales máximos propuestos son naturalmente más altos que el tipo impositivo marginal máximo actual del 35\%. Los resultados obtenidos concuerdan con los de Fairfield para Chile y la Argentina, pues ese autor propone tipos impositivos óptimos de entre el 55\% y el 64\% para Chile y entre el 56\% y el 59\% para la Argentina, asumiendo elasticidades similares a las de los Estados Unidos. En el caso de Francia, Saez (2001) utilizó elasticidades del ingreso imponible para proponer un tipo impositivo óptimo para las personas de altos ingresos del $75 \%$. 


\section{Conclusiones}

En este trabajo se estudió la relación entre la política tributaria y la desigualdad de los ingresos en el Ecuador y se obtuvieron varios resultados empíricos. En primer lugar, el impuesto sobre la renta de las personas físicas en el Ecuador es, en líneas generales, progresivo, pues los índices de progresividad de Kakwani y Suits y el análisis de la curva de concentración muestran que las personas más ricas generalmente pagan más impuestos sobre la renta que las más pobres. Sin embargo, al analizar los tipos impositivos efectivos pagados por las personas de muy altos ingresos, se encontró que estos disminuían entre los grupos de ingresos más altos, especialmente entre el 0,1\%, el 0,05\%, el 0,01\% y el 0,001\% más ricos de la población.

En segundo lugar, la capacidad redistributiva del impuesto sobre la renta de las personas físicas es muy débil. El índice de redistribución de Reynolds-Smolensky sugiere que la desigualdad de los ingresos es solo un punto menor después del impuesto sobre la renta que antes de este, al disminuir de 0,66 a 0,65 en 2008 y de 0,64 a 0,63 en 2010. Que la capacidad redistributiva sea tan pequeña se debe sobre todo a las deducciones del impuesto sobre la renta de las personas físicas, que son aprovechadas sobre todo por las personas de altos ingresos.

En tercer lugar, los resultados obtenidos mediante el enfoque de la curva de concentración mostraron que los contribuyentes que más se beneficiaron de las deducciones del impuesto sobre la renta de las personas físicas en el período 2008-2010 fueron aquellos de los percentiles octogésimo y superiores de la base de datos fiscal (que corresponden al $5 \%$ más rico cuando se emplea una variable de control para la población total). Las personas de altos ingresos tienen mayores probabilidades de reducir su ingreso imponible mediante deducciones legales, erosionando de ese modo la base imponible y anulando el efecto distributivo buscado por la reforma tributaria de 2008. Los resultados del ejercicio de microsimulación estático realizado muestran que el efecto redistributivo del impuesto sobre la renta de las personas físicas podría ser mayor si las deducciones fiscales estuvieran mejor dirigidas y controladas.

En cuarto lugar, a pesar de la inversión de la tendencia en 2010, los tipos impositivos efectivos medios pagados por las personas de altos ingresos son muy bajos cuando los dividendos forman parte integral de la base del impuesto sobre la renta de las personas físicas. El grupo de ingresos correspondiente al $1 \%$ más rico pagó un tipo impositivo efectivo medio del $7 \%$ y el $0,1 \%$ más rico un tipo del 9,4\% en todo el período examinado.

En quinto lugar, se recurrió a la literatura sobre elasticidad del ingreso imponible para obtener tipos impositivos óptimos para las personas de altos ingresos. Si bien el tipo impositivo marginal máximo en el Ecuador es actualmente del 35\%, los resultados obtenidos sugieren que el tipo máximo óptimo podría situarse entre el 57\% y el 63\%.

Por último, es importante subrayar la necesidad fundamental de cerrar las lagunas fiscales existentes, no obstante los esfuerzos realizados por el Servicio de Rentas Internas del Ecuador en los últimos años.

Las nuevas deducciones del impuesto sobre la renta de las personas físicas, que se introdujeron para incrementar la equidad tributaria, son un instrumento demasiado contundente y los encargados de la formulación de políticas deberían considerar otras maneras de modificar la carga fiscal de los contribuyentes de bajos y medianos ingresos. Asimismo, deberían mejorar la orientación de las deducciones del impuesto sobre la renta en la cima de la distribución del ingreso.

Los resultados obtenidos también sugieren que se podría crear una estructura más progresiva para el impuesto sobre la renta de las personas físicas en el Ecuador e incrementar el tipo impositivo marginal máximo. Naturalmente, existe la preocupación de que un aumento de los tipos impositivos 
marginales máximos podría afectar las tasas de trabajo y creación de empresas y, en consecuencia, el crecimiento económico. Sin embargo, la investigación empírica (Piketty, Saez y Stantcheva, 2014) ha probado que algunos países, como los Estados Unidos y el Reino Unido, donde los tipos impositivos marginales máximos se redujeron considerablemente con el paso del tiempo, no han crecido más rápidamente que los países donde se han mantenido tipos impositivos altos. Sin embargo, la desigualdad de los altos ingresos ha aumentado. Así, una mayor progresividad tributaria en el Ecuador llevaría a un incremento en la recaudación tributaria y la inversión pública, en particular en educación, aptitudes y atención sanitaria.

\section{Bibliografía}

Alvaredo, F. (2010), "The rich in Argentina over the Twentieth Century, 1932-2004", Top Incomes a Global Perspective, A. B. Atkinson y T. Piketty, Oxford, Oxford University Press.

Alvaredo, F. y J. Londoño (2013), "High incomes and personal taxation in a developing economy: Colombia 1993-2010", CEQ Working Paper Series, № 12, Commitment to Equity.

Amarante, V. y otros (2011), "Distributive impacts of alternative tax structures. The case of Uruguay", Documentos de Trabajo, № 09/11, Montevideo, Universidad de la República.

Atkinson, A. B. (2014), "After Piketty?", The British Journal of Sociology, vol. 65, No 4, Wiley.

Atkinson, A. B. y T. Piketty (2010), Top Incomes: a Global Perspective, Oxford, Oxford University Press. (2007), Top Incomes over the Twentieth Century. A Contrast between Continental European and Englishspeaking Countries, Oxford, Oxford University Press.

Atkinson, A. B., T. Piketty y E. Saez (2011), "Top incomes in the long run of history", Journal of Economic Literature, vol. 49, No 1, Nashville, Tennessee, American Economic Association.

Auten, G. y R. Carroll (1999), "The effect of income taxes on household income", Review of Economics and Statistics, vol. 81, № 4, Cambridge, Massachusetts, The MIT Press.

Bourguignon, F. y A. Spadaro (2006), "Microsimulation as a tool for evaluating redistribution policies", Journal of Economic Inequality, vol. 4, № 1, Springer.

Burdín, G., F. Esponda y A. Vigorito (2014), "Inequality and top incomes in Uruguay: a comparison between household surveys and income tax micro-data", CEQ Working Paper Series, № 1, Commitment to Equity.

Cano, L. (2015), "Income Inequality, Top Income Shares and Economic Mobility: Ecuador 2004-2011", tesis, Toulouse, Universidad de Toulouse 1 Capitole.

CEPAL (Comisión Económica para América Latina y el Caribe) (2014), Panorama Social de América Latina 2014 (LC/G.2635-P), Santiago.

(2013), Panorama Social de América Latina 2013 (LC/G.2580), Santiago. (2012), Panorama Social de América Latina 2012 (LC/G.2557-P), Santiago.

Cetrángolo, O. y J. Gómez Sabaini (2006), Tributación en América Latina: en busca de una nueva agenda de reformas, Libros de la CEPAL, № 93 (LC/G.2324-P), Santiago, Comisión Económica para América Latina y el Caribe (CEPAL).

Chetty, R. (2009), "Is the taxable income elasticity sufficient to calculate deadweight loss? The implications of evasion and avoidance", American Economic Journal: Economic Policy, vol. 1, № 2, Nashville, Tennessee, American Economic Association.

Cornia, A. (ed.) (2014), Falling Inequality in Latin America. Policy Change and Lessons, Oxford University Press. (2010), "Income distribution under Latin America's new left regimes", Journal of Human Development and Capabilities, vol. 11, № 1, Taylor and Francis.

Cornia, A., J. Gómez Sabaini y B. Martorano (2011), "A new fiscal pact, tax policy changes and income inequality: Latin America during the last decade", UNU-WIDER Working Paper, № 70, Helsinki, Instituto Mundial de Investigaciones de Economía del Desarrollo (UNU-WIDER).

Diamond, P. y E. Saez (2011), "The case for a progressive tax: from basic research to policy recommendations", Journal of Economic Perspectives, vol. 25, № 4, Nashville, Tennessee, American Economic Association.

Fairfield, T. (2010), The Politics of Revenue-Raising Tax Reform in Latin America, Berkeley, Universidad de California.

Fairfield, T. y M. Jorratt (2016), "Top income shares, business profits, and effective tax rates in contemporary Chile", Review of Income and Wealth, vol. 62, N S1, Wiley. 
Feldstein, M. (1999), "Tax avoidance and the deadweight loss of the income tax", Review of Economics and Statistics, vol. 81, № 4, Cambridge, Massachusetts, The MIT Press.

Figari, F., A. Paulus y H. Sutherland (2015), "Microsimulation and policy analysis", Handbook of Income Distribution, vol. 2, A. B. Atkinson y F. Bourguignon, Amsterdam, Elsevier.

Gasparini, L. y otros (2009), "A turning point? Recent developments on inequality in Latin America and the Caribbean", CEDLAS Working Papers, № 081, Universidad Nacional de la Plata.

Gómez Sabaini, J. (2006), "Cohesión social, equidad y tributación. Análisis y perspectivas para América Latina", serie Políticas Sociales, № 127 (LC/L.2641-P), Santiago, Comisión Económica para América Latina y el Caribe (CEPAL).

Gómez Sabaini, J. y J. Jiménez (2012), "Tax structure and tax evasion in Latin America", serie Macroeconomía del Desarrollo, № 118 (LC/L.3455), Santiago, Comisión Económica para América Latina y el Caribe (CEPAL).

Gómez Sabaini, J. y D. Moran (2013), "Política tributaria en América Latina: agenda para una segunda generación de reformas”, serie Macroeconomía del Desarrollo, № 133 (LC/L.3632), Santiago, Comisión Económica para América Latina y el Caribe (CEPAL).

González, I. y R. Martner (2009), "Del síndrome del casillero vacío al desarrollo inclusivo: buscando los determinantes de la distribución del ingreso en América Latina", Documento de Trabajo, Santiago, Comisión Económica para América Latina y el Caribe (CEPAL).

Goñi, E., H. López y L. Servén (2011), "Fiscal redistribution and income inequality in Latin America", World Development, vol. 39, № 9, Amsterdam, Elsevier.

Gruber, J. y E. Saez (2002), "The elasticity of taxable income: evidence and implications", Journal of Public Economics, vol. 84, № 2, Amsterdam, Elsevier.

Hanni, M., R. Martner y A. Podestá (2015), "El potencial redistributivo de la fiscalidad en América Latina", Revista CEPAL, Nº 116 (LC/G.2643-P), Santiago, Comisión Económica para América Latina y el Caribe (CEPAL).

Jiménez, J. P., J. C. Gómez Sabaini y A. Podestá (2010), "Tax gap and equity in Latin America and the Caribbean", Fiscal Studies, № 16, Santiago, Comisión Económica para América Latina y el Caribe (CEPAL).

Kakwani, N. (1977), "Measurement of tax progressivity: an international comparison", The Economic Journal, vol. 87, N 345, Royal Economic Society.

Landais, C., T. Piketty y E. Saez (2011), Pour une révolution fiscale: un impôt sur le revenu pour le XXlème siècle, París, Seuil.

Lindsey, L. (1987), "Individual taxpayer response to tax cuts: 1982-1984, with implications for the revenue maximizing tax rate", Journal of Public Economics, vol. 33, № 2, Amsterdam, Elsevier.

López-Calva, L. y N. Lustig (2010), Declining Inequality in Latin America: A Decade of Progress, Washington, D.C., Brookings Institution Press.

Lustig, N., L. López-Calva y E. Ortiz-Juárez (2013), "Deconstructing the decline in inequality in Latin America", Policy Research Working Paper, № 6552, Washington, D.C., Banco Mundial.

OCDE/CEPAL/CIAT/BID (Organización de Cooperación y Desarrollo Económicos/Comisión Económica para América Latina y el Caribe/Centro Interamericano de Administraciones Tributarias/Banco Interamericano de Desarrollo) (2015), Revenue Statistics in Latin America and the Caribbean, 1990-2013, París, OECD Publishing.

Piketty, T. (2015), "Putting distribution back at the center of economics: reflections on capital in the TwentyFirst Century", The Journal of Economic Perspectives, vol. 29, № 1, Nashville, Tennessee, American Economic Association.

Piketty, T. (2014), El capital en el siglo XXI, Madrid, Fondo de Cultura Económica. (2003), "Income inequality in France, 1901-1998", Journal of Political Economy, vol. 111, № 5, Chicago, The University of Chicago Press.

_(2001), Les hauts revenus en France au XXe siècle: inégalités et redistributions, 1901-1998, París, Bernard Grasset.

Piketty, T. y E. Saez (2003), "Income inequality in the United States, 1993-1998", Quarterly Journal of Economics, vol. 118, № 1, Oxford University Press.

Piketty, T., E. Saez e I. Stantcheva (2014), "Optimal taxation of top labor incomes: a tale of three elasticities", American Economic Journal, vol. 6, № 1, Nashville, Tennessee, American Economic Association.

Poterba, J. (2007), "Income inequality and income taxation", Journal of Policy Modeling, vol. 29, № 4, Amsterdam, Elsevier.

Rawlings, L. y G. Rubio (2005), "Evaluating the impact of conditional cash transfer programs", The World Bank Economic Review, vol. 20, № 1, Oxford, Oxford University Press. 
Reynolds, M. y E. Smolensky (1977), Public Expenditures, Taxes and the Distribution of Income: the United States 1950-1961, Nueva York, Academic Press.

Riihela, M., R. Sullstrom y M. Tuomala (2014), "Top incomes and top tax rates: implications for optimal taxation of top incomes in Finland", Tampere Economic Working Papers Net Series, № 88, Universidad de Tampere.

Roca, J. (2009), "Tributación directa en Ecuador: evasión, equidad y desafíos de diseño", serie Macroeconomía del Desarrollo, № 85 (LC/L.3057-P), Santiago, Comisión Económica para América Latina y el Caribe (CEPAL).

Saez, E. (2001), "Using elasticities to derive optimal income tax rates", The Review of Economic Studies, vol. 68, No 1, Oxford, Oxford University Press.

Saez, E., J. Slemrod y S. Giertz (2012), "The elasticity of taxable income with respect to marginal tax rates: a critical review", Journal of Economic Literature, vol. 50, № 1, Nashville, Tennessee, American Economic Association.

Saez, E. y M.R. Veall (2005), "The evolution of high incomes in Northern America: lessons from Canadian evidence", The American Economic Review, vol. 95, № 3, Nashville, Tennessee, American Economic Association.

Spadaro, A. (2007), Microsimulation as a Tool for the Evaluation of Public Policies. Methods and Applications, Bilbao, Fundación BBVA.

Suits, D. (1977), "Measurement of tax progressivity", American Economic Review, vol. 67, № 4, Nashville, Tennessee, American Economic Association.

Tanzi, V. (2013), Tax Reform in Latin America: a Long Term Assessment", Washington, D.C., Woodrow Wilson International for Scholars.

Van Kerm, P. y A. Peichl (2007), "Progres: Stata module to measure distributive effects of an income tax", Statistical Software Components, № S456867, Boston College. 


\section{Anexo A1}

Gráfico A1.1

Ecuador: contribuyentes como proporción del total de unidades tributarias (adultos de 20 o más años de edad, 2004-2011

(En porcentajes)

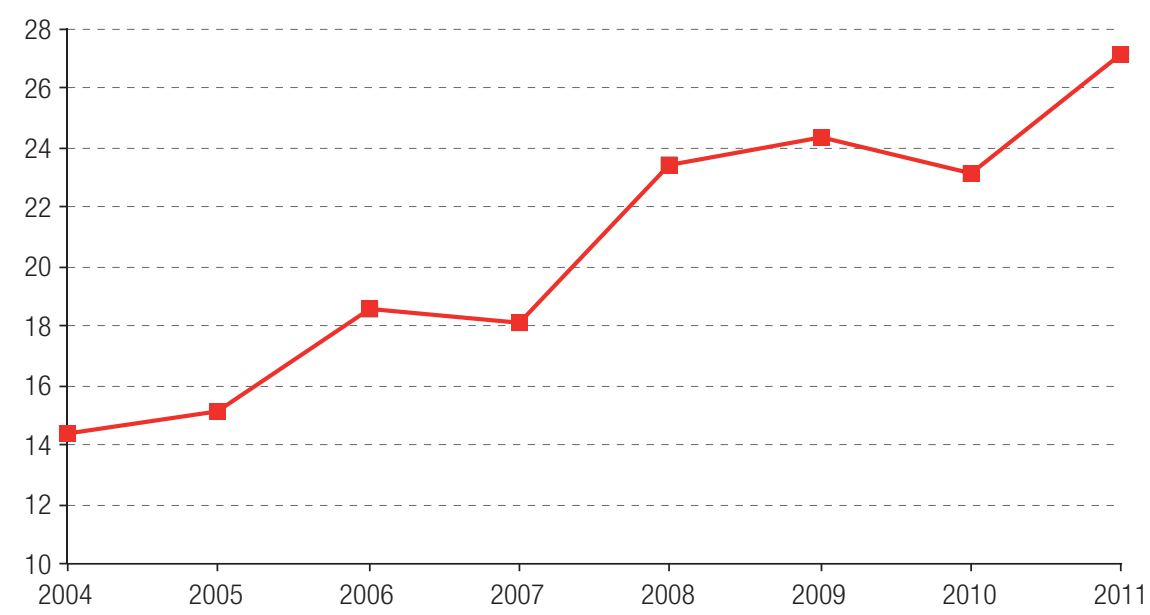

Fuente: Elaboración propia, sobre la base de datos de declaraciones de la renta individuales del Servicio de Rentas Internas (SRI) del Ecuador y estimaciones de población del Instituto Nacional de Estadística y Censos (INEC). 